This item was submitted to Loughborough's Research Repository by the author.

Items in Figshare are protected by copyright, with all rights reserved, unless otherwise indicated.

\title{
A reinforcement learning-based user-assisted caching strategy for dynamic content library in small cell networks
}

PLEASE CITE THE PUBLISHED VERSION

https://doi.org/10.1109/TCOMM.2020.2977895

PUBLISHER

IEEE-INST ELECTRICAL ELECTRONICS ENGINEERS INC

VERSION

AM (Accepted Manuscript)

\section{PUBLISHER STATEMENT}

Personal use of this material is permitted. Permission from IEEE must be obtained for all other uses, in any current or future media, including reprinting/republishing this material for advertising or promotional purposes, creating new collective works, for resale or redistribution to servers or lists, or reuse of any copyrighted component of this work in other works

\section{LICENCE}

\section{All Rights Reserved}

\section{REPOSITORY RECORD}

Zhang, Xinruo, Gan Zheng, Sangarapillai Lambotharan, Mohammad Reza Nakhai, and Kai-Kit Wong. 2020. "A Reinforcement Learning-based User-assisted Caching Strategy for Dynamic Content Library in Small Cell Networks". Loughborough University. https://hdl.handle.net/2134/13244531.v1. 


\title{
A Reinforcement Learning-Based Caching Strategy for Dynamic Content Library in Small Cell Networks
}

\author{
Xinruo Zhang, Member, IEEE, Gan Zheng, Senior Member, IEEE, \\ Sangarapillai Lambotharan, Senior Member, IEEE, and Mohammad Reza Nakhai, Senior Member, IEEE
}

\begin{abstract}
This paper studies the problem of joint edge cache placement and content delivery in cache-enabled small cell networks in the presence of spatio-temporal content dynamics unknown a priori, where small base stations (SBSs) satisfy users' content requests either directly from their local caches, or by retrieving from other SBSs' caches or from the content server. In contrast to previous approaches that assume a static content library at the server, this paper considers a more realistic nonstationary content library, where new contents may be emerged over time at different locations. To keep tracling the dynamic content library, we propose that the new contents cached at users can be exploited by the SBSs to timely update their flexible cache memories priom to their routine off-peak main cache updates from the content server. To account for the variations in traffic demands as well as the limited caching space at the SBSs, a reinforcement learning based user aided caching algorithm is developed to asymptotically optimize the caching policy with the target of maximizing the weighted network utility in the long run. Simulation results verify the superior performance of the proposed caching strategy against various benchmark designs.
\end{abstract}

Index Terms—non-stationary bandit; cache placement; content delivery; time-varying popularity; dynamic content library

\section{INTRODUCTION}

Global mobile data traffic is growing at an unprecedented rate and is predicted to account for more than 63 percent of total data traffic, reaching 48.3 Exabytes per month by 2021 [1]. The content delivery network (CDN) that has been widely adopted for traffic congestion reduction, is expected to carry 71 percent of all internet traffic by 2021, of which 82 percent will be video traffic. However, the backhaul data rate demand between base stations (BSs) and the core network incurred by such rapid traffic growth, especially during peak traffic periods, has become the major revenue and technical bottleneck for the network operators [2]. Due to the fact that a large portion of backhaul traffic is contributed by transmitting duplicate popular data from the core network to multiple users [3], caching popular contents, e.g., video, social media, news and maps, that are repeatedly requested by a large number of users in local memories installed at BSs to eliminate duplicate data transmission, has recently attracted significant attention of researchers [4]. The integration of content caching with

Xinruo Zhang, Gan Zheng and Sangarapillai Lambotharan are with Wolfson School of Mechanical, Electrical and Manufacturing Engineering, Loughborough University, LE11 3TU, U.K. (e-mail: \{x.zhang, g.zheng, s.lambotharan\}@lboro.ac.uk).

Mohammad Reza Nakhai is with Centre for Telecommunications Research, King's College London, WC2B 4BG, U.K. (e-mail: reza.nakhai@kcl.ac.uk). small base stations (SBSs) that provide short-range, low-power and low-cost transmission underlaying the existing macrocell cellular networks, allows popular mobile data to be prefetched from the core network during off-peak traffic hours and to be delivered to edge users at peak times. Such integration provides opportunities not only to offload the backhaul traffic load, but also to improve system performance such as energy efficiency and transmission delay, and hence, significantly alleviate the backhaul and latency bottlenecks in conventional wireless CDN [5]. Considering the fact that the capacity of cache storage is highly limited at the individual SBSs as compared to the massive content library at the content server, efficient caching mechanisms are advocated to be developed for the network operators to maximally benefit from caching techniques. Recently, cooperative caching and joint optimization of different caching locations, e.g., central cloud caching and SBSs caching, has been proposed as a potential solution to the enhancement of content caching performance in dynamic mobile networks [2]. By coordinating content caching at different locations, the individual SBSs may cache differentiated contents and retrieve the requested content from other cache locations at a lower cost rather than from the content server. However, provided that the individual SBSs can only observe the instantaneous content requests of their users, the content popularity distribution and/or users' preference may be unknown a priori and may vary with time and locations. Hence, a timely estimation of users' content requests is challenging but essential for the effective caching policy design as well as for the reliable and cost-efficient operation of networks under the uncertainty of traffic demands.

\section{A. Related Works}

Most approaches in the literature assume finite cache storage with time-invariant content popularity distribution perfectly known at BSs [4]-[14], and design either content placement strategies [5]-[10] or content delivery strategies [11]-[14] in various network scenarios. Joint consideration of content placement and delivery strategies have been studied in recent years based on either coded [15]-[18] or uncoded [19]-[22] data. The assumption of content popularity distribution known at BSs, nevertheless, is not realistic in practical scenarios. In recent years, using machine learning method to predict the unknown content popularity, and proactively cache the popular contents at the BSs in advance of users' requests, 
has attracted the attention of the researchers [23]. In [24], the authors propose a Lyapunov optimization approach to hybrid content caching design to tackle spatial dynamics in traffic demands, where the content popularity is not required. The authors in [2] and [25] relax this assumption and introduce the multi-armed bandit (MAB) based learning approaches to estimate the content popularity distribution over time horizon. However, [2] only considers spatial diversity of static content popularity, whilst [25] assumes unknown and time-invariant content popularity. The authors in [26] model the cache replacement problem as a Markov decision process and propose a Q-learning algorithm to trade-off the global and local popularity demands in heterogeneous networks. Assuming a Poisson request model, [27] develops a transfer learning based approach with a finite training time to improve the estimation of the content popularity in a heterogenous network based on a training set of ratings. This work, nevertheless, deals with content caching only in a single BS for one period in time, while an online learning approach may be more suitable for the estimation of content popularity over the time horizon. The authors in [28] propose a regret learning based per-BS caching strategy to learn the spatio-temporal traffic demands and to capture the trade-off of the local and the global content popularity. However, the aforementioned works simply ignore the fact that the contents can be dynamic over time: new contents are constantly introduced to the content library and their popularity distributions change over time. For instance, the popularity of some contents such as news vanishes within a limited time whilst others such as music and movies may attract sustained requests for a long time. Hence, those works without considering the dynamic content library in the nature of their designs, may not be able to catch up with the rapid variations of the content demands in practice. The authors in [29] propose an ON-OFF traffic model to capture the impact of dynamic contents on cache performance based on Che's approximation, whereas, they have sacrificed the key fact that the request processes at different caches are independent.

\section{B. Contributions}

This paper focuses on joint design of edge cache placement and content delivery in small cell networks. In contrast to the existing caching designs that assume stationary content library and/or time-invariant content popularity, we consider a non-stationary content library with spatio-temporal content dynamics unknown a priori. The novel contribution of this paper is the development of a reinforcement learning based user aided caching algorithm that keeps tracking the dynamic content library and aims at maximizing the averaged weighted network utility in the long run. The main contributions of this paper are summarized as follows:

We explore users' caches to improve caching performance at the SBSs based on the fact that some users may have cached new contents through other networks, for example wireless local area networks (WLAN). To be specific, a portion of the cache unit at each SBS is allocated as the flexible cache memory, which can be timely updated with the new contents cached at users prior to the routine off-peak main cache update from the content server.

A non-stationary bandit problem inspired user aided foresighted caching algorithm is developed to adaptively track the spatio-temporal variations of users' content demands and gradually optimize the content caching and delivery policy in the long run.

We consider three phases of different time scales for joint cache placement and content delivery in small cell networks. Phase I is the content delivery phase at the individual time slots, where the content demand of each user is satisfied from one of the caching locations with various serving gains. Phase II is the SBSs' flexible cache update phase, where the flexible caches of the SBSs can be more frequently updated with users' cached new contents. Phase III is the SBSs' main cache update phase at off-peak times, where the cache units of the SBSs are updated from the content server.

To account for the limited caching spaces at the SBSs, content caching coordination is employed among SBSs and the near-optimal constrained cross-entropy (CCE) method is adopted in Phase III to solve the cache placement optimization problem with low-complexity.

\section{Organization and Notations}

The rest of this paper is organized as follows. Section II introduces the system model and the assumptions. In section III, the joint caching placement and content delivery problem is formulated and then transformed to a reinforcement learning assisted content placement optimization problem that can be solved via the near-optimal CCE method. In section IV, a bandit-inspired user aided caching algorithm is proposed to cope with the spatio-temporal content dynamics. Numerical simulation results are presented and analyzed in section $\mathrm{V}$. Finally, section VI concludes the paper.

Notations: Throughout the paper, $w, \mathbf{w}$ and $\mathbf{W}$, respectively, indicate a scalar $w$, a vector $\mathbf{w}$ and a matrix $\mathbf{W} . \mathbb{E}(\cdot)$ is the expected value, $\mathbb{B}^{n} m$ denotes the binary space of $n$-by- $m$ matrices and $\mathbb{C N}(0,1)$ is the zero mean complex Gaussian random variables with unit variance. $\left\|_{.}\right\|_{0}$ is the $l_{0}$-norm indicating the number of non-zero entries in the vector, and $I_{\mathrm{f} . \mathrm{g}}$ is an indicator function that returns one if $\{\cdot\}$ holds true and zero otherwise.

\section{SYSTEM MODEL}

\section{A. System Scenario}

As illustrated in Fig. 1, we consider a time-slotted downlink small cell network consisting of $N_{b}$ SBSs serving their respective $N_{u}$ users over a shared spectrum. Let us denote by $\mathcal{L}_{b}=\left\{1, \cdots, N_{b}\right\}, \mathcal{L}_{u}^{b}=\left\{1, \cdots, N_{u}\right\}$ and $\mathcal{T}_{1}=\{1, \cdots, T\}$, respectively, the index set of SBSs, the index set of users associated with SBS $b$, and the index set of discrete time slots. Featuring cache units, the individual SBSs are connected with each other via inter-SBS links and to the content server via capacity-limited fronthaul and backhaul links. A central processing unit $(\mathrm{CU})$ coordinates all content caching and 

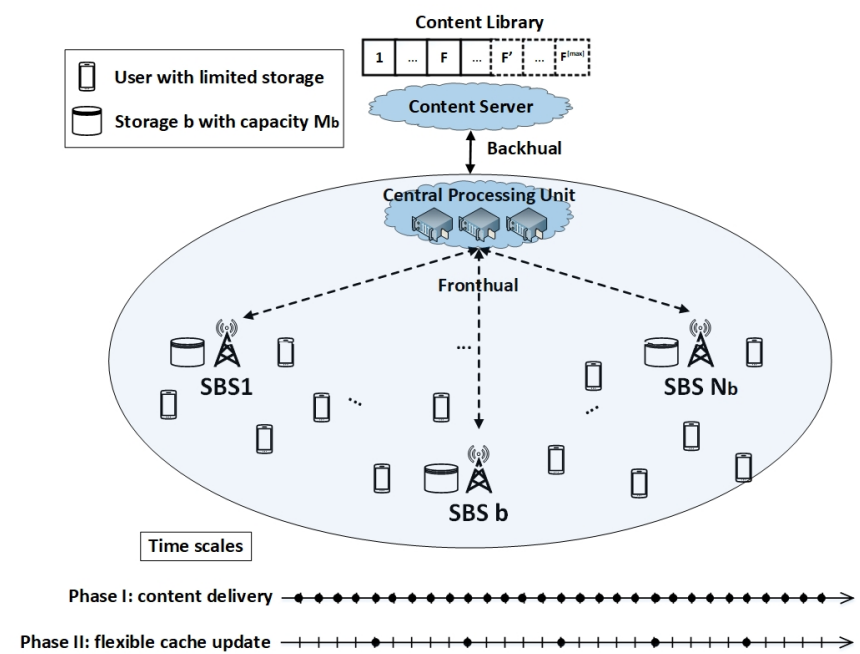

Fig. 1. Illustration of the system scenario and three phases of different time scales for joint cache placement and content delivery.

delivery strategies for SBSs based on perfect knowledge of channel state information. The notations in this paper are listed in Table I.

1) Non-stationary Content Library: Let us consider a realistic scenario, where the new contents are constantly introduced into the system and the finite content library at the content server is thus non-stationary. Let us denote by $\mathcal{F}=\left\{1, \cdots, F, \cdots, F^{[\max ]}\right\}$ the finite content library with individual content sizes of $\left\{S_{f}\right\}_{f 2 \mathrm{~F}}$, where $F$ and $F^{[\max ]}$, respectively, denote the initial and the maximum number of contents in the content library. Let us denote by $\mathcal{F}^{t}=$ $\left\{1, \cdots, F^{0}\right\}$ the content library at the $t$-th time slot, $t \in \mathcal{T}_{1}$, where $F^{0}$ indicates the current number of contents in the library. It is evident that $F \leq F^{0} \leq F^{[\max ]}$. In case the content library has $F^{0}=F^{[\max ]}$ available contents at time slot $t$ whilst the new contents are continuously emerging with time, the least frequently used contents at the content server will be evicted and replaced by the newly emerged contents. Note that the dynamic content library adopted in the considered scenario naturally results in the time-varying content popularity, which is unknown to the SBSs.

2) New Contents at Users: Each individual user is equipped with a capacity-limited local cache memory and can only cache one content at each time. At the end of each time slot, each user randomly updates its cache memory with one of its requested contents. In addition, newly emerged contents may be cached at some users either via being generated by the local users themselves, or by being brought in through other networks such as WLAN or due to users' mobility. The users are motivated to upload these potentially popular new contents to their associated SBSs for the incentive payment. From the perspective of the SBSs, let us denote by $\kappa^{\text {[user] }}$ the per-unit per-time-slot equivalent cost of the users' uploading incentives, which covers the costs such as for allocating extra bandwidth for users' contents uploading.

3) Users' Content Demands: At each time slot $t, t \in \mathcal{T}_{1}$, user $u, u \in \mathcal{L}_{u}^{b}$, may request $N_{f}^{u}$ number of contents that
TABLE I

NOTATION

\begin{tabular}{|c|c|}
\hline Symbol & Definition \\
\hline $\mathcal{L}_{b}$ & Index set of SBSs \\
\hline $\mathcal{L}_{u}^{b}$ & Index set of users associated with SBS $b$ \\
\hline $\mathcal{T}_{1}$ & Index set of discrete time slots in Phase I \\
\hline $\mathcal{T}_{2}$ & Index set of $\left\{\tau^{[\mathrm{flex}]}\right\}$ in Phase II \\
\hline $\mathcal{T}_{3}$ & Index set of $\left\{\tau^{[\text {main] }}\right\}$ in Phase III \\
\hline $\mathcal{F}$ & Index set of finite content library at the server \\
\hline $\mathcal{F}^{t}$ & Index set of current content library at time $t$ \\
\hline$S_{f}$ & Size of content $f$ \\
\hline$N_{f}^{u}$ & Number of content requests by user $u$ at each time slot \\
\hline$N_{f}^{\lfloor\text {new }\rfloor}$ & Number of new contents added to the content server in Phase II \\
\hline$\{b\}_{b 2 L_{b}}$ & The spatial shifts of content popularity among individual SBSs \\
\hline$\left\{\begin{array}{c}t \\
b, f\end{array}\right\}_{f 2 \mathrm{~F}^{t}}$ & The unknown content popularity distribution at SBS $b$ at time $t$ \\
\hline $\mathbf{d}_{u}^{t}$ & Content demand vector of user $u$ at time $t$ \\
\hline$d_{u f}^{t}$ & Whether the content $f$ is requested by user $u$ at time $t$ \\
\hline$\pi^{[\text {local }]}$ & $\begin{array}{l}\text { Per-unit gross serving gain of an SBS serving users from } \\
\text { its local cache }\end{array}$ \\
\hline$\pi^{[\mathrm{SBS}]}$ & $\begin{array}{l}\text { Per-unit gross serving gain of an SBS serving users by fetching } \\
\text { content from other SBSs' caches }\end{array}$ \\
\hline$\pi^{\text {[server] }}$ & $\begin{array}{l}\text { Per-unit gross serving gain of an SBS serving users by fetching } \\
\text { content from the content server }\end{array}$ \\
\hline$\kappa^{[\text {user] }}$ & Per-unit per-time-slot equivalent cost of users' uploading incentives \\
\hline$M_{b}$ & Capacity of the cache unit at SBS $b$ \\
\hline$\xi$ & Portion for flexible cache memory at each SBS \\
\hline $\mathbf{c}_{p}^{t}$ & Content caching placement policy at time $t$ \\
\hline$c_{b, f}^{t}$ & Whether content $f$ is cached at SBS $b$ at time $t$ \\
\hline $\mathbf{c}_{r}^{t}$ & Content retrieving policy at time $t$ \\
\hline$c_{b, b^{\prime}, f}^{t}$ & Whether content $f$ is retrieved by SBS $b$ from SBS $b^{0}$ at time $t$ \\
\hline$c_{b, s, f}^{t}$ & Whether content $f$ is retrieved by SBS $b$ from server at time $t$ \\
\hline $\mathbf{c}_{u}^{t}$ & Content uploading policy at time $t$ \\
\hline$c_{b, u, f}^{t}$ & Whether content $f$ is uploaded from user $u$ to SBS $b$ at time $t$ \\
\hline$G_{b, f}^{u, t}$ & $\begin{array}{l}\text { Net serving gain for SBS } b \text { serving user } u \text { with content } f \\
\text { directly from its local cache at time } t\end{array}$ \\
\hline$G_{b, s, f}^{u, t}$ & $\begin{array}{l}\text { Net serving gain for SBS } b \text { serving user } u \text { by fetching } \\
\text { content } f \text { from the content server at time } t\end{array}$ \\
\hline$G_{b, b^{\prime}, f}^{u, t}$ & $\begin{array}{l}\text { Net serving gain for SBS } b \text { serving user } u \text { by fetching } \\
\text { content } f \text { from SBS } b^{0} \text { at time } t\end{array}$ \\
\hline$C_{b, u, f}^{t}$ & Cost of SBS $b$ updating content $f$ from user $u$ at time $t$ \\
\hline $\mathcal{R}_{b}\left(d_{u f}^{t}\right)$ & $\begin{array}{l}\text { Instantaneous serving reward for SBS } b \text { serving user } u \\
\text { with content } f \text { at time } t\end{array}$ \\
\hline $\mathbf{\Upsilon}^{t}$ & Joint reward distribution of contents for all SBSs at time $t$ \\
\hline$\beta$ & The discount factor in Algorithm 2 \\
\hline$\epsilon^{t}$ & The exploration/exploitation trade-off for Phase II in Algorithm 2 \\
\hline$\Phi_{b}^{0}$ & Mixed recent number of content requests \\
\hline$\mu^{t}$ & The global/local trade-off for Phase II in Algorithm 2 \\
\hline
\end{tabular}

are not cached by itself from its associated SBS $b$. Let the user $u$ 's content demands at time $t$ be denoted by $\mathbf{d}_{u}^{t}=$ $\left\{d_{u 1}^{t}, \cdots, d_{u f}^{t}, \cdots, d_{u F^{\prime}}^{t}\right\}$, where the binary scalar $d_{u f}^{t} \in$ $\{0,1\}$ indicates whether or not the content $f$ is requested by user $u$, and $\left\|\mathbf{d}_{u}^{t}\right\|_{0}=N_{f}^{u}$. In addition to the temporal variability of the content popularity, we further consider the spatial diversity of the content popularity distribution among individual SBSs, i.e., the users associated with different SBSs may have different preference for the contents. Let us denote by $\Delta_{b}$ the shift of the content popularity distribution at SBS $b$ and denote by $\left\{\Theta_{b, f}^{t}\right\}_{f 2 \mathrm{~F} t}$ the actual dynamic content popularity that is unknown at SBS $b$. The instantaneous content demands of the users, i.e., $\left\{\mathbf{d}_{u}^{t}\right\}_{u 2 \mathrm{~L}_{u}}$, will be satisfied either by directly from the local cache of the serving SBS, or by 
retrieving from one of the caches of the other SBSs or from the content server with different per-unit gross serving gains of $\pi^{[\text {local }]}, \pi^{[\mathrm{SBS}]}$ and $\pi^{[\mathrm{server}]}$, respectively. For simplicity, we assume normalized and identical $\pi^{[\text {local }]}, \pi^{[\mathrm{SBS}]}, \pi^{[\text {[server] }}$ and $\kappa^{\text {[user] }} \in[0,1]$ at all SBSs. To take into account the backhaul traffic offloading as well as to fully utilize the cache units at the SBSs, let us assume $\pi^{[\text {server }]}<\pi^{[\mathrm{SBS}]}<\pi^{[\text {local] }}$.

4) Cache Units at the SBSs: Each individual SBS is equipped with a cache unit with capacity of $M_{b}, b \in \mathcal{L}_{b}$. To fully exploit the contents cached at the local users, a portion with capacity of $\xi M_{b}$ of each cache unit is allocated as the flexible cache memory. The flexible cache memory that is made up of expensive and high-speed static random access memory (RAM), can be timely updated from the caches of the local users, whilst the remainder of the cache unit that is made up of cheaper and slower RAM, will be updated at a more infrequent pace, for instance, from the content server during off-peak traffic hours.

5) Three Phases of Different Time Scales: Three phases of different time scales, namely, Phase I: the content delivery phase; Phase II: the SBSs' flexible cache update phase; and Phase III: the SBSs' main cache update phase are proposed for joint caching placement and content delivery in this paper. Recall that we have denoted by $\mathcal{T}_{1}=\{1, \cdots, T\}$ the index set of discrete time slots for Phase I. Similarly, let us denote by $\mathcal{T}_{2}$ and $\mathcal{T}_{3}$, respectively, the index sets of $\left\{\tau^{[\text {flex }]}\right\}$ for Phase II and $\left\{\tau^{[\text {main }]}\right\}$ for Phase III, where $\tau^{[\text {flex }]}$ and $\tau^{[\text {main }]}$ are the respective time scales of Phase II and Phase III.

In Phase I, the SBSs satisfy the instantaneous content requests of their users, and update the corresponding reward information to the $\mathrm{CU}$ at each time slot $t, t \in \mathcal{T}_{1}$. In Phase II, i.e., for every $\tau^{[\text {flex }]}$ time slots, $N_{f}^{[\text {new }]}$ number of new contents are added to the content server and might be cached by some users. Each user broadcasts its cached content directory to its associated SBS, and the SBS will then make a decision on whether or not to update its flexible cache with the user's cached content.

In Phase III, i.e., for every $\tau^{[\text {main] }}$ time slots, the CU designs cache placement policy for the SBSs and dispatchs the contents from the content server via backhaul links to update SBSs' cache units.

\section{B. Downlink Transmission}

Recall that a set $\mathcal{L}_{u}^{b}$ of local users are associated with SBS $b$ in the considered small cell network. Let us denote by $\Psi_{b u}^{t}$ the channel gain between SBS $b$ and user $u$ at the $t$-th time slot, and denote by $P_{b}^{[\mathrm{Tx}]}$ the transmit power of SBS $b$. The signalto-interference-plus-noise ratio (SINR) for user $u$ served by SBS $b$ at time slot $t$, is given by

$$
\operatorname{SINR}_{b u}^{t}=\frac{P_{b}^{[\mathrm{Tx}]}\left|\Psi_{b u}^{t}\right|^{2}}{\sum_{b^{\prime} 2 \mathrm{~L}_{b}, b^{\prime} € b} P_{b^{\prime}}^{[\mathrm{Tx}]}\left|\Psi_{b^{\prime} u}^{t}\right|^{2}+\sigma_{u}^{2}},
$$

where $\sigma_{u}^{2}$ is the noise variance of the additive white Gaussian noise at user $u$. Then, with the normalized bandwidth, the instantaneous downlink data rate for user $u$ served by SBS $b$ at time slot $t$, can be expressed as

$$
R_{b u}^{t}=\log _{2}\left(1+\operatorname{SINR}_{b u}^{t}\right) .
$$

\section{Content Caching and Retrieving}

Let us define the binary vector $\mathbf{c}_{p}^{t}=\left\{c_{b, f}^{t} \in\{0,1\}, \forall b \in\right.$ $\left.\mathcal{L}_{b}, f \in \mathcal{F}^{t}\right\}$ as the content caching policy at time $t$, where $c_{b, f}^{t}=1$ and $c_{b, f}^{t}=0$ indicate that the content $f$ is cached and is not cached at SBS $b$, respectively. This caching policy will be designed in Phase III for SBSs' main cache update, and, might be updated in Phase II for the portion of flexible cache memory. Let us denote by $\mathbf{c}_{r}^{t}=\left\{c_{b, b^{\prime}, f}^{t}, c_{b, s, f}^{t} \in\{0,1\}, \forall b^{0} \neq\right.$ $\left.b, b \in \mathcal{L}_{b}, b^{0} \in \mathcal{L}_{b}, f \in \mathcal{F}^{t}\right\}$ the content retrieving policy at time $t$, where $c_{b, b^{\prime}, f}^{t}=1$ and $c_{b, b^{\prime}, f}^{t}=0$ indicate that the content $f$ is fetched and is not fetched by SBS $b$ from SBS $b^{0}$, respectively. $c_{b, s, f}^{t} \in\{0,1\}$ denotes whether or not the content $f$ is retrieved from the content server by SBS $b$ at time $t$. Clearly, the user demand of content $f, f \in \mathcal{F}^{t}$ at each time slot $t, t \in \mathcal{T}_{1}$, will be satisfied by serving either from the content server or from one of the SBSs, as

$$
c_{b, s, f}^{t}+\sum_{b^{\prime} 2 \mathrm{~L}_{b}} c_{b, b^{\prime}, f}^{t}=1 .
$$

Let us denote the content uploading policy at time $t$ as $\mathbf{c}_{u}^{t}=$ $\left\{c_{b, u, f}^{t} \in\{0,1\}, \forall b \in \mathcal{L}_{b}, u \in \mathcal{L}_{u}^{b}, f \in \mathcal{F}^{t}\right\}$, where $c_{b, u, f}^{t}=1$ and $c_{b, u, f}^{t}=0$, respectively, represent that the content $f$ is uploaded and is not uploaded from user $u$ to SBS $b$. The pertime-slot equivalent cost of SBS $b$ for the incentive payment of content $f$ uploaded from user $u, u \in \mathcal{L}_{u}^{b}$, is defined as

$$
C_{b, u, f}^{t}=\kappa^{\text {[user] }} S_{f} c_{b, u, f}^{t}, \forall t \in \mathcal{T}_{1}, b \in \mathcal{L}_{b}, f \in \mathcal{F}^{t} .
$$

Then, the net serving gain of SBS $b$ storing content $f$ and serving user $u$ at time slot $t$, can be defined as

$$
\begin{array}{r}
G_{b, f}^{u, t}=\pi^{[\text {local }]} S_{f} d_{u f}^{t} c_{b, f}^{t}-\sum_{u^{\prime} \boxminus u, u^{\prime} 2 \mathrm{~L}_{u}^{b}} C_{b, u^{\prime}, f}^{t}, \\
\forall t \in \mathcal{T}_{1}, u \in \mathcal{L}_{u}^{b}, b \in \mathcal{L}_{b}, f \in \mathcal{F}^{t},
\end{array}
$$

where the terms at the right hand side of (5) denote, respectively, the gross serving gain for SBS $b$ serving user $u$ with content $f$ directly from its local cache, and the per-time-slot equivalent cost if the content $f$ cached at SBS $b$ is uploaded from the other local users. The net serving gains of SBS $b$ for serving user $u$ by retrieving content $f$ from SBS $b^{0}$ and from the content server at time slot $t$, respectively, are given by

$$
\begin{gathered}
G_{b, b^{\prime}, f}^{u, t}=\pi^{[\mathrm{SBS}]} S_{f} d_{u f}^{t} c_{b, b^{\prime}, f}^{t}-\sum_{u^{\prime} 2 \mathrm{~L}_{u}^{b^{\prime}}} C_{b^{\prime}, u^{\prime}, f}^{t}, \\
\forall b^{0} \neq b, b \in \mathcal{L}_{b}, b^{0} \in \mathcal{L}_{b}, u \in \mathcal{L}_{u}^{b}, t \in \mathcal{T}_{1}, f \in \mathcal{F}^{t}, \\
G_{b, s, f}^{u, t}=\pi^{[\text {[server] }} S_{f} d_{u f}^{t} c_{b, s, f}^{t}, \forall t \in \mathcal{T}_{1}, u \in \mathcal{L}_{u}^{b}, b \in \mathcal{L}_{b}, f \in \mathcal{F}^{t},
\end{gathered}
$$

where (6) indicates that if the content $f$ cached at SBS $b^{0}$ and retrieved by SBS $b$ is uploaded from the local users of SBS $b^{0}$, the corresponding cost need to be subtracted. Per time slot $t$, the content demand $f$ of user $u$ is satisfied from one of the locations with one of the net serving gains of $\left\{G_{b, f}^{u, t}, G_{b, b^{\prime}, f}^{u, t}, G_{b, s, f}^{u, t}\right\}$. The instantaneous serving reward, i.e., 
the weighted data rate, of SBS $b$ for serving user $u$ with content $f$ at time slot $t$, can be defined as

$$
\begin{aligned}
& \mathcal{R}_{b}\left(d_{u f}^{t}\right)=\left(G_{b, f}^{u, t}+G_{b, b^{\prime}, f}^{u, t}+G_{b, s, f}^{u, t}\right) R_{b u}^{t}, \\
& \quad \forall b^{0} \neq b, b \in \mathcal{L}_{b}, b^{0} \in \mathcal{L}_{b}, u \in \mathcal{L}_{u}^{b}, f \in \mathcal{F}^{t}, t \in \mathcal{T}_{1} .
\end{aligned}
$$

Note that (8) jointly considers the caching hit ratio, the backhaul traffic offloading and the content delivery by assigning different weighting factors, i.e., $G_{b, f}^{u, t}, G_{b, b^{\prime}, f}^{u, t}$, or $G_{b, s, f}^{u, t}$, to the transmission data rate. This reward will be useful in designing cache placement policy as well as content delivery policy in the subsequent sections.

\section{PROBLEM FORMULATION AND OPTIMIZATION}

\section{A. Problem Formulation}

Let us denote by $\mathbf{w}^{t}=\left\{\mathbf{c}_{p}^{t}, \mathbf{c}_{r}^{t}, \mathbf{c}_{u}^{t}\right\}$ the joint content caching, retrieving and delivery policy of the SBSs at time slot $t$. The objective of the CU is to design a joint caching policy $\left\{\mathbf{w}^{t}\right\}$ to offload as much traffic as possible from the content server, whilst ensuring the quality of service (QoS) of the users. Hence, the problem of interest can be formulated as the maximization of the long-term average reward of the network, i.e., the average weighted network utility, as

$$
\begin{array}{ll} 
& \max _{\mathbf{f} \mathbf{w}^{t} \mathbf{g}}\left\{\lim _{T !} \frac{1}{T} \sum_{t 2 \mathrm{~T}_{1}} \sum_{b 2 \mathrm{~L}_{b}} \sum_{f 2 \mathrm{~F}^{t}} \sum_{u 2 \mathrm{~L}_{u}^{b}} \mathcal{R}_{b}\left(d_{u f}^{t}\right)\right\} \\
\text { s.t. } \quad & \mathrm{C} 1: \sum_{f 2 \mathrm{~F}^{t}} S_{f} c_{b, f}^{t} \leq M_{b}, \forall b \in \mathcal{L}_{b}, t \in \mathcal{T}_{1}, \\
& \mathrm{C} 2: c_{b, s, f}^{t}+\sum_{b^{\prime} 2 \mathrm{~L}_{b}} c_{b, b^{\prime}, f}^{t}=1, \forall b \in \mathcal{L}_{b}, f \in \mathcal{F}^{t}, t \in \mathcal{T}_{1}, \\
& \mathrm{C} 3: c_{b, b^{\prime}, f}^{t} \leq c_{b^{\prime}, f}, \forall b \neq b^{0}, b, b^{0} \in \mathcal{L}_{b}, f \in \mathcal{F}^{t}, t \in \mathcal{T}_{1}, \\
& \mathrm{C} 4: c_{b, s, f}^{t} \in\{0,1\}, \forall b \in \mathcal{L}_{b}, f \in \mathcal{F}^{t}, t \in \mathcal{T}_{1} . \\
\mathrm{C} 5: c_{b, b^{\prime}, f}^{t} \in\{0,1\}, \forall b \neq b^{0}, b, b^{0} \in \mathcal{L}_{b}, f \in \mathcal{F}^{t}, t \in \mathcal{T}_{1} . \\
\mathrm{C} 6: c_{b, u, f}^{t} \in\{0,1\}, \forall b \in \mathcal{L}_{b}, f \in \mathcal{F}^{t}, u \in \mathcal{L}_{u}^{b}, t \in \mathcal{T}_{1}, \\
\mathrm{C} 7: c_{b, f}^{t} \in\{0,1\}, \forall b \in \mathcal{L}_{b}, f \in \mathcal{F}^{t}, t \in \mathcal{T}_{1} .
\end{array}
$$

$\tau^{\text {[flex] }}$ time slots, and design $\left\{\mathbf{c}_{p}^{t}\right\}$ at every $\tau^{[\text {main] }}$ time slots, respectively. Let us denote by $\boldsymbol{\Upsilon}^{t}=\left\{v_{b, f}^{t}, \forall b \in \mathcal{L}_{b}, f \in \mathcal{F}^{t}\right\}$ the joint reward distribution over the non-stationary library of contents, where $v_{b, f}^{t}=\mathbb{E}\left[d_{u f}^{t} R_{b u}^{t}\right]$. Then, we can define the expected overall reward among all $\mathrm{S} \equiv$ as

$$
\begin{aligned}
& \mathcal{S}\left(\mathbf{c}_{p}^{t}\right)=\mathbb{E}\left[\sum_{b 2 \mathrm{~L}_{b}} \sum_{f 2 \mathrm{~F}^{t}} \sum_{u 2 \mathrm{~L}_{u}^{b}} \mathcal{R}_{b}\left(d_{u f}^{t}\right)\right]=\sum_{b 2 \mathrm{~L}_{b}} \sum_{f 2 \mathrm{~F}^{t}} S_{f} N_{u} \Upsilon_{b, f}^{t} \\
& {\left[\left(\pi^{[\text {local] }}-\kappa^{\text {[user] }} \sum_{u^{\prime} 2 \mathrm{~L}_{u}^{b}, u^{\prime} \boldsymbol{G} u} c_{b, u^{\prime}, f}^{t}\right) c_{b, f}^{t}+\pi^{[\text {server] }}\left(1-\max _{b 2 \mathrm{~L}_{b}} c_{b, f}^{t}\right)\right.} \\
& \left.+\max _{b^{\prime} 2 \mathrm{~L}_{b}, b^{\prime} \boxminus b}\left(\left(\pi^{[\mathrm{SBS}]}-\kappa^{[\mathrm{user}]} \sum_{u^{\prime} 2 \mathrm{~L}_{u}^{b^{\prime}}} c_{b^{\prime}, u^{\prime}, f}^{t}\right) c_{b^{\prime}, f}^{t}\left(1-c_{b, f}^{t}\right)\right)\right],
\end{aligned}
$$

where the terms at the right hand side of (10) indicate, respectively, that the user will be directly served if its associated SBS caches the requested content, the user will be served from the content server if no SBS caches the requested content, and the user will be served by retrieving content from one of the other SBSs associated with the highest serving reward if its associated SBS caches no requested content.

The main content caching policy, i.e., $\left\{\mathbf{c}_{p}^{t}\right\}$, will be designed based on the learning result of $\boldsymbol{\Upsilon}^{t}$ at every $\tau^{\text {[main] time slots in }}$ Phase III of the proposed algorithm via the following content placement optimization problem, as

$$
\begin{array}{ll} 
& \max _{\mathbf{c}_{p}^{t}} \mathcal{S}\left(\mathbf{c}_{p}^{t}\right) \\
\text { s.t. } \quad & \mathrm{C} 1: \sum_{f 2 \mathrm{~F}^{t}} S_{f} c_{b, f}^{t} \leq M_{b}, \forall b \in \mathcal{L}_{b}, \\
& \mathrm{C} 2: c_{b, f}^{t} \in\{0,1\}, \forall b \in \mathcal{L}_{b}, f \in \mathcal{F}^{t} .
\end{array}
$$

\section{B. Constrained Cross-Entropy Method for Optimization}

Problem in (11) can be regarded as a knapsack problem in combinatorial optimization with weights of $\left\{S_{f}\right\}_{f 2 \mathrm{~F}^{t}}$, and can be optimally solved via branching algorithms such as the branch and bound $(\mathrm{B} \& \mathrm{~B})$ algorithm, or via the semidefinite relaxation (SDR) approach [2]. Instead of solving problem (11) via the B\&B algorithm or the SDR approach with high computational complexity, we solve (11) with a near-optimal solution $\mathbf{c}_{p}^{t}$ via the low-complexity CCE method. The crossentropy (CE) method is an adaptive variance minimization algorithm for estimating probabilities of rare events and the principle outcome is the construction of a random sequence of solutions which converges probabilistically to the optimal or near-optimal solution [30]. However, the original CE method for unconstrained optimization cannot be applied directly to (11) in the presence of constraints, as many sample points might not be in the feasible region. Consequently, we adopt the CCE method with the penalty approach [31] to solve the problem in (11), which relaxes the constraints in a similar fashion of the Lagrangian relaxation and penalizes the evaluation 


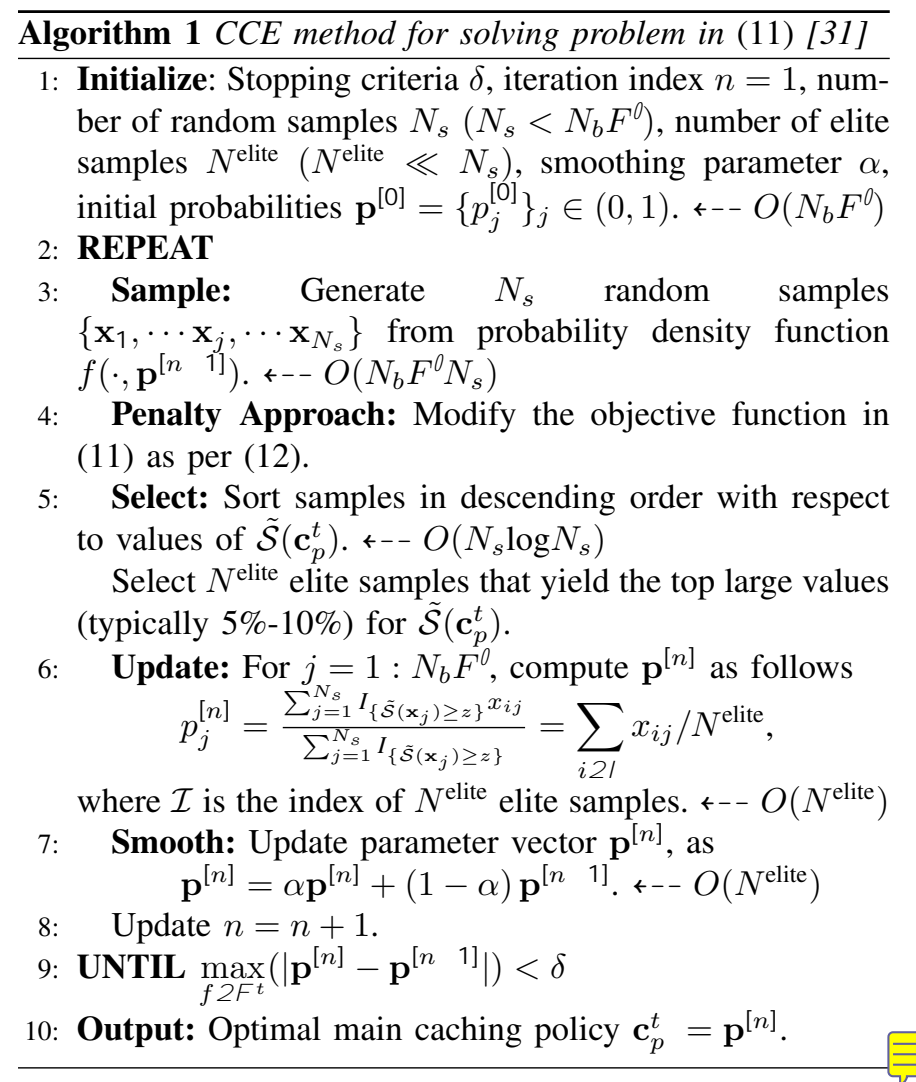

of infeasible solutions. Let us modify the objective function and relax the constraints in (11), as

$$
\begin{aligned}
z & =\max _{\mathbf{c}_{p}^{t} 2 \mathrm{C}} \tilde{\mathcal{S}}\left(\mathbf{c}_{p}^{t}\right) \\
& =\mathcal{S}\left(\mathbf{c}_{p}^{t}\right)+\sum_{b 2 \mathrm{~L}_{b}} H_{b} \max \left(\sum_{f 2 \mathrm{~F} t} S_{f} c_{b, f}^{t}-M_{b}, 0\right),
\end{aligned}
$$

where the penalty parameter $H_{b} \gg 0$ indicates the importance of the penalty function and $\mathcal{C} \subset \mathbb{B}^{N_{b} F^{\prime}}$ is the feasible region. The steps and the computational complexity of each step of the CCE method are detailed in Algorithm 1. The CCE method associates a stochastic estimation problem, i.e.,

$$
\mathbb{P}\left(\tilde{\mathcal{S}}\left(\mathbf{c}_{p}^{t}\right) \geq z\right)=\sum_{\mathbf{c}_{p}^{t} 2 \mathrm{C}} I_{\mathrm{f} S\left(\mathbf{c}_{p}^{t}\right) \quad z \mathrm{~g}} f\left(\mathbf{c}_{p}^{t}, \mathbf{p}\right),
$$

where $I_{\mathrm{f} S\left(\mathbf{c}_{p}^{t}\right) \quad z \mathrm{~g}}$ is an indicator function with value one if $\tilde{\mathcal{S}}\left(\mathbf{c}_{p}^{t}\right) \geq z$ and 0 otherwise, and $f\left(\mathbf{c}_{p}^{t}, \mathbf{p}\right)$ is a Bernoulli distribution characterized by a parameter vector $\mathbf{p}$, as

$$
f(\mathbf{x}, \mathbf{p})=\prod_{j=1}^{n}\left(p_{j}\right)^{x_{j}}\left(1-p_{j}\right)^{1} x_{j}, x_{j} \in\{0,1\}, j=1, \cdots, n .
$$

Let us define $z$ as the worst value of $\tilde{\mathcal{S}}\left(\mathbf{c}_{p}^{t}\right)$ among $N^{\text {elite }}$ elite samples. The CCE method aims at estimating $\mathbb{P}\left(\tilde{\mathcal{S}}\left(\mathbf{c}_{p}^{t}\right) \geq z\right)$ with increasing threshold value of $z$, converging either to the global optimum $z$ or a value close to it via importance sampling. At each iteration $n$, the new value of $z$ is used to update $\mathbf{p}^{[n]}$, whilst the new vector $\mathbf{p}^{[n]}$ in turn, is used for generating better samples as per steps 6 and 3 of Algorithm 1 , respectively. The application of the smoothing parameter $\alpha(0.4 \leq \alpha \leq 0.9)$ in step 7 is to prevent the occurrences of all zeros or all ones sub-optimal solutions and the convergence to the degenerate case will have a polynomial rather than exponential speed [31].

\section{The Proposed Foresighted CAChing Algorithm}

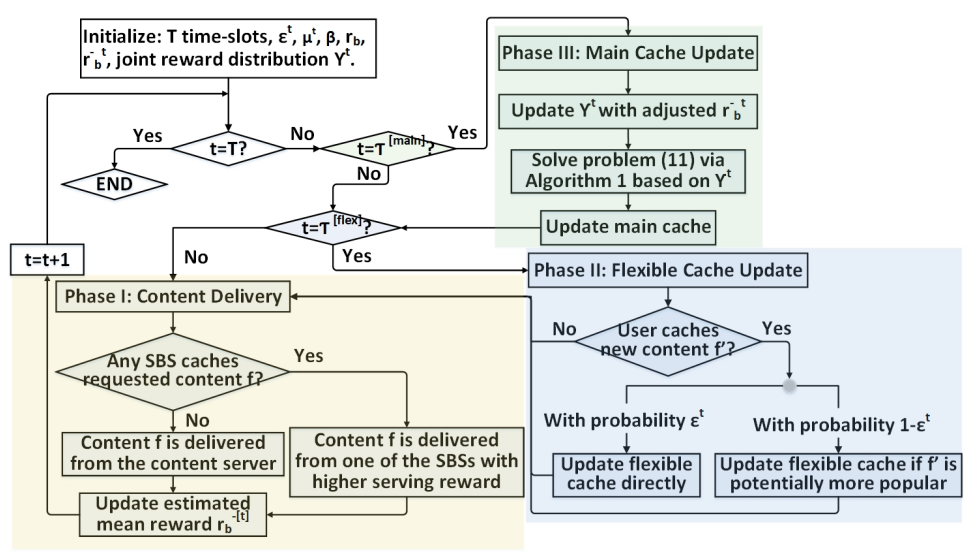

Fig. 2. Flowchart of the proposed caching strategy.

In this section, the proposed user aided foresighted caching lgorithm will be introduced to account for the traffic offloadIng and caching hits while ensuring the QoS of the users. The main objective of the proposed caching strategy is to progressively optimize the caching policy time slot by time slot, so as to maximize the long-term average reward, i.e., the average weighted network utility. Let us consider a nonstationary generalization of the MAB problem that models a system of $F^{0}$ arms whose expected rewards are independent and identically distributed over time with unknown means and may vary across time. The objective of the MAB problem is to maximize the accumulated reward over time via exploring the environment to find profitable arms, while exploiting current knowledge to make the empirically best decisions among a set of arms [33]. The caching problem studied in this paper can be modelled as a non-stationary bandit problem, where the SBSs can be regarded as the agents, $F^{0}$ arms correspond to the current library of $F^{0}$ contents at the content server, and the associated reward of playing an arm can be defined as the instantaneous reward for satisfying users' content demands, as per (8). The details of the proposed foresighted caching strategy are described in Algorithm 2 and Fig. 2, where three phases of different time scales for joint content caching and delivery are proposed and are explained below:

1) Phase I: At each time slot $t, t \in \mathcal{T}_{1}$, the instantaneous content requests of the users are received and satisfied according to the delivery policy. Then, the rewards of the individual requested contents are updated at the CU.

2) Phase II: For every $\tau^{[\mathrm{flex}]}$ time slots, $\tau^{[\mathrm{flex}]} \in \mathcal{T}_{2}$, the contents associated with the lowest reward in SBSs' flexible caches will be replaced by the new contents cached at the users accordingly. To be specific, with the probability of $\epsilon^{t}$, we explore new contents cached by local users and update SBSs' flexible caches directly. With the probability of $1-\epsilon^{t}$, the flexible cache will only be updated with the more potentially 


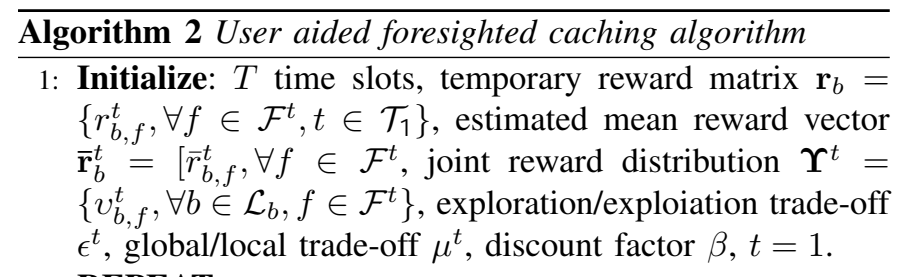

2: REPEAT

3: If $t=\tau^{\text {[main] }}$, Phase III. Main Cache Placement

4: The CU updates joint reward distribution $\boldsymbol{\Upsilon}^{t}$, as $v_{b, f}^{t}=$ $\bar{r}_{b, f}^{t}+\sum_{t^{\prime}=1}^{t} \sum_{u 2 \mathrm{~L}_{u}^{b}} \beta^{\left(t \quad t^{\prime}\right)} S_{f} d_{u f}^{\left[t^{\prime}\right]} \sqrt{\frac{2 \log n_{t}}{T_{b, f}}}$, where $T_{b, f}=$ $\sum_{t^{\prime}=1}^{t} \beta^{(t}{ }^{\left.t^{\prime}\right)} I_{\mathrm{f} c c_{b, f}^{\left[t^{\prime}\right]}=1 \mathrm{~g}}$ is the discounted number of times content $f$ has been cached by SBS $b$ and $n_{t}=\sum_{f 2 \mathrm{~F}^{t}} T_{b, f}$.

5: Design cache placement policy via Algorithm 1 and update SBSs' cache units in a sorted order based on $\boldsymbol{\Upsilon}^{t}$.

6: End If

7: If $t=\tau^{[\mathrm{flex}]}$, Phase II. Flexible Cache Update

8: Each SBS $b$ observes its local users' cached contents.

9: If A new content $f^{0}$ is cached by its local user $u$

10: -with probability $\epsilon^{t}$, update SBS $b$ 's flexible cache directly;

11: -with probability $1-\epsilon^{t}$, update $\boldsymbol{\Phi}_{b}^{0}$ as per (15), and update the flexible cache only if $\boldsymbol{\Phi}_{b}^{0}\left(f^{0}\right)$ is larger than that of the contents in flexible cache of SBS $b$.

12: End if

13: End If

14: Phase I. Content Delivery at Each Time Slot $t$

15: Each SBS $b$ receives its user demands $\left\{\mathbf{d}_{u}^{t}\right\}_{u 2 L_{u}^{b}}$.

16: If the requested content $f \in \mathcal{F}^{t}$ is cached at $\operatorname{SBS} b$ -SBS $b$ serves the user directly from its local cache.

Else If the requested content $f$ is cached at other SBSs -SBS $b$ serves the user by fetching content $f$ from one of the other SBSs associated with the higher serving reward. Else If no SBS caches the requested content $f$

-SBS $b$ serves the user by fetching from the content server. End if

17: Update $\left\{\mathbf{r}_{b}\right\}_{b}$ as $r_{b, f}^{t}=\sum_{u 2 \mathrm{~L}_{u}^{b}} \mathcal{R}_{b}\left(d_{u f}^{t}\right), \forall f \in \mathcal{F}^{t}, b \in \mathcal{L}_{b}$.

18: Update estimated mean reward as $\overline{\mathbf{r}}_{b}^{t}=\frac{\sum_{t^{\prime}=1}^{t} \mathbf{r}_{b} \beta^{\left(t-t^{\prime}\right)}}{t}$.

19: Update $t=t+1$.

20: UNTIL $t=T$

popular new contents, based on the mixed recent number of content requests $\boldsymbol{\Phi}_{b}^{0}$, given by

$$
\boldsymbol{\Phi}_{b}^{0}=\left(1-\mu^{t}\right) \boldsymbol{\Phi}_{c}+\mu^{t} \boldsymbol{\Phi}_{b}, b \in \mathcal{L}_{b},
$$

where $\boldsymbol{\Phi}_{c}=\sum_{b 2 \mathrm{~L}_{b}} \boldsymbol{\Phi}_{b}$ and $\boldsymbol{\Phi}_{b}=\left\{\Phi_{b, f}, \forall b \in \mathcal{L}_{b}, f \in \mathcal{F}^{t}\right\}$, respectively, denote the global, i.e., among all SBSs, and the local, i.e., for a particular SBS $b$, recent numbers of user requests of the individual contents. The global/local trade-off $\mu^{t}, 0 \leq \mu^{t} \leq 1$, is employed to capture the impact of content caching coordination among SBSs.

3) Phase III: For every $\tau^{[\text {main }]}$ time slots, $\tau^{[\text {main }]} \in \mathcal{T}_{3}$, a perturbation procedure is applied to the estimated mean reward $\overline{\mathbf{r}}_{b}^{t}$ according to step 5 in Algorithm 2. Such adjustment implements a trade-off between exploring the contents that are not frequently cached and may yield a better accumulated reward in the future by artificially increasing their estimated mean reward, and exploiting the arms associated with the highest mean reward so far based on the past observations. Then, the CU optimizes cache placement policy based on $\Upsilon^{t}$ via Algorithm 1 and updates SBSs' cache units.

\section{A. Computational Complexity Analysis}

The computational burden of the proposed algorithm mainly lies in designing the cache placement policy in step 5 of Algorithm 2 via Algorithm 1. As stated in the previous section, the optimal or near optimal solution of problem in (11) can be found via the B\&B algorithm, the SDR approach [2] or the CCE method. The worst case complexity of the B\&B algorithm is $O\left(F^{0 \sum_{b \in \mathcal{L}_{b}} M_{b}}\right)$, which is the same as that of the exhaustive search [25]. The SDR approach relaxes problem in (11) as a semidefinite programming problem, which can be solved via the interior-point algorithm with a worst case computational complexity of $O\left(\max \left\{\tau_{s}, N_{b}\left(F^{0}+1\right)\right\}^{4} \tau_{s}^{0.5} \log \left(\frac{1}{\sigma}\right)\right)$ [2], where $\sigma$ is the solution accuracy and $\tau_{s}$ denotes the problem size of (11). In addition, a recovery approach is necessary to recover the rank-one solution and to reconstruct the optimal caching decisions $\mathbf{c}_{p}^{t}$, which will further increase the computational complexity of the problem. The overall computational complexity of the CCE method in Algorithm 1 is $O\left(N_{b} F^{0} M_{b} N_{s} \log N_{s}\right)$, and the main complexity is due to the performance evaluation of $N_{s}$ samples for the modified objective functions $\tilde{\mathcal{S}}\left(\mathbf{c}_{p}^{t}\right)$ as per step 5 of Algorithm 1. Thus, the complexity is low and can be further reduced through a trade-off between the complexity and solution accuracy.

\section{SIMULATION RESULTS}

Consider a downlink small cell network comprising 3 SBSs, where each individual SBS serves $N_{u}=4$ randomly deployed users. The non-stationary content library has an initial library of $F=250$ contents and a finite capacity of $F^{[\max ]}=300$. Each individual content size varies as $S_{f} \in\{1,2\}$ and the capacity of the caching unit at each SBS is $M_{b}=15$ with $\xi=0.2$. The Phase III for SBSs' main cache update and the Phase II for flexible cache update, respectively, occur at every $\tau^{[\text {main }]}=8$ time slots and at every $\tau^{[\text {flex }]}=3$ time slots. For every $\tau^{[\text {flex }]}=3$ time slots, $N_{f}^{[\text {new }]}=2$ new contents will be added to the content server and might be cached by some random users. The per-unit gross gains for SBSs to serve users directly from their local caches, by fetching from caches of the other SBSs and by retrieving from the content server are, respectively, $\pi^{[\text {local }]}=1, \pi^{[\mathrm{SBS}]}=0.5$ and $\pi^{\text {[server] }}=0.1$, whilst the per-unit per-time-slot equivalent cost of users' uploading incentives is $\kappa^{\text {[user] }}=0.1$. The commonly used Zipf distribution [2], [6], given by

$$
\Theta_{b, f !}^{t} \quad{ }_{b}=\frac{f^{\gamma^{t}}}{\sum_{f=1}^{F^{\prime}} f \gamma^{t}}, f \in \mathcal{F}^{t},
$$

is adopted to model the actual content popularity distribution of the dynamic content library that is unknown to the SBSs, 
where $\gamma^{t}=2$ is the Zipf exponent indicating the popularity skewness, and the shifts of content popularity at the individual SBSs are $\left\{\Delta_{b}\right\}=\{0,4,8\}$. Each user has $N_{f}^{u}=3$ content requests at the individual time slots generated according to the actual content popularity distribution $\left\{\Theta_{b, f}^{t}\right\}_{f 2 \mathrm{~F}}{ }^{t}$. Note that we employ the Zipf distribution in the simulation just as an illustration to evaluate our proposed caching algorithm, and the choice of the content popularity distribution model will not affect the effectiveness of our proposed algorithm. The channel gain is modelled as $\Psi_{b u}^{t}=\mathbf{h}_{b u}^{t 2} G_{a} L_{b u} \sigma_{F}^{2} e^{0.5 \frac{\left(\sigma_{s} \ln 10\right)^{2}}{100}}$ [34], where $\mathbf{h}_{b u}^{t} \sim \mathbb{C N}(0,1), G_{a}=15 \mathrm{dBi}$ denotes the antenna gain, $L_{b u}(\mathrm{~dB})=128.1+37.6 \log _{10}(\ell)$ [35] is the path loss model over a distance of $\ell \mathrm{km}$ between SBS $b$ and user $u$, $\sigma_{s}=10 \mathrm{~dB}$ represents log-normal shadowing standard deviation and $\sigma_{F}^{2}=1$ is the variance of complex Gaussian fading coefficient. The other simulation parameters are described, unless otherwise stated, as follows: transmit poser $P_{b}^{[\mathrm{Tx}]}=23$ $\mathrm{dBm}, N=100$ random samples, $N^{\text {elite }}=10$ elite samples, smoothing parameter $\alpha=0.9$, exploration/exploitation tradeoff $\epsilon^{t}=0.8$, discount factor $\beta=0.93$ and global/local trade-off $\mu^{t}=0.7$. The proposed strategy is evaluated with $T=1000$ time slots for each set of parameter setting. Three designs that consider no user cache are chosen as the benchmark designs, namely, the algorithm in [2], the algorithm in [25] and the random caching design. For fair comparison, identical constraints have been applied to all strategies.

1) Benchmark design in [2]: The benchmark design in [2] estimates the content popularity distribution via the standard upper confidence bound (UCB) algorithm [33]. The estimated mean reward is set as the estimated popularity distribution, given by

$$
\bar{\theta}_{b, f}^{t}=\frac{O_{b, f}^{t}}{N_{b, f}^{t}},
$$

where $O_{b, f}^{t}$ and $N_{b, f}^{t}$ denote, respectively, the long-term observation of the request number of content $f$ in SBS $b$, and the total number of time slots the requests of content $f$ are satisfied by local SBSs' caches.

2) Benchmark design in [25]: The benchmark design in [25] learns the content popularity distribution via the combinatorial UCB algorithm based on the past observation of the content demands of the users. The instantaneous reward associated with file $f$ is defined as $r_{b, f}^{t}=\sum_{u 2 \mathrm{~L}_{u}} S_{f} d_{u f}^{t}$, and the estimated mean reward is given by

$$
\bar{\theta}_{b, f}^{t}=\frac{\sum_{t^{\prime}=1}^{t} r_{b, f}^{t^{\prime}}}{T_{b, f}},
$$

where $T_{b, f}$ is the number of times content $f$ has been cached by SBS $b$. For fair comparison, the benchmark designs in [2] and [25] have been embedded with the sliding-window [36], which emphasizes more on the recent observations, so as to better adapt to our considered scenario.

3) Random caching design: In Phase III, the random caching design randomly caches contents at the individual SBSs without considering any content caching coordination among SBSs. This design is employed to indicate the lower bound and to demonstrate the advantage of the cooperative caching and joint optimization of different caching locations.

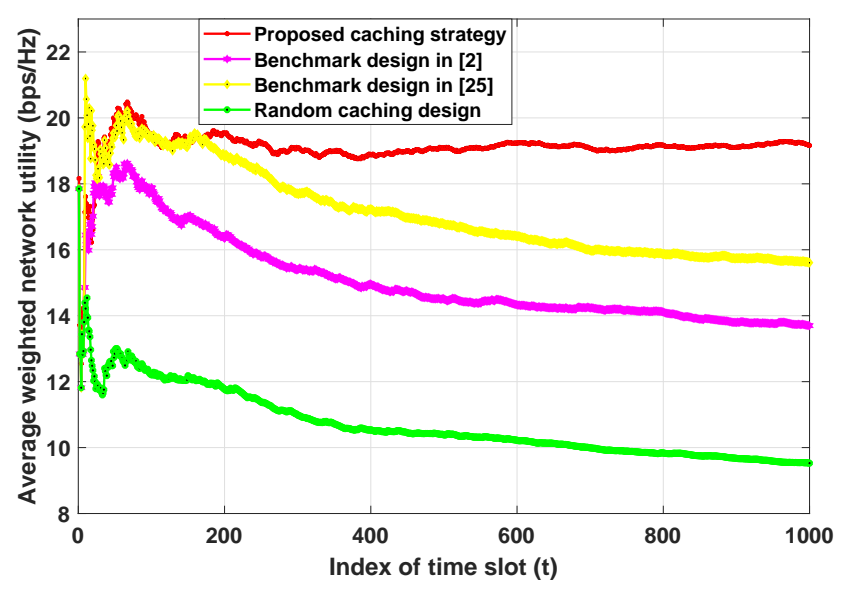

Fig. 3. Comparison of average weighted network utility for different strategies at individual time slots.

Fig. 3 illustrates the comparison of the average reward, i.e., the weighted network utility, of the proposed foresighted caching strategy against the benchmark designs in [2], [25] and the random caching design at the individual time slots. As can be observed from Fig. 3, the proposed strategy outperforms all benchmark designs and the performance gap increases with the increasing number of time slots. This is due to the negligence of both the evolution of the content library and the potentiality of user caches in the nature of designs in [2] and [25]. Furthermore, it is obvious that the random caching design has the worst performance among all the strategies. The reason is that it simply caches contents randomly at the individual SBSs without involving any content caching coordination among SBSs or any learning process to estimate the unknown variations in user demands. In contrast, the proposed strategy takes into account the spatio-temporal variations in users' content demands, and maximally benefits from the user caches through timely updating the SBS's flexible cache with the potentially more popular new contents cached by users, thus provides better adaptation to the user demand variations.

Fig. 4 provides an illustration of the evolution of content placement policy of SBS 1 at the $1^{\text {st }}$, the $8^{\text {th }}$, the $15^{\text {th }}$, the $25^{\text {th }}$ and the $30^{\text {th }}$ iterations of Algorithm 1 at the $10^{\text {th }}$ time slot. It is clear from the figure that by updating and smoothing the parameter vector $\mathbf{p}^{[n]}$ as per step 6 and step 7 in Algorithm 1 , respectively, better random samples can be produced in the subsequent iterations. Furthermore, the CCE method in Algorithm 1 converges within approximately 30 iterations and the outputs at the $25^{\text {th }}$ iteration are close to the converged solutions, which indicates a much lower complexity and a faster convergence speed as compared to the B\&B algorithm.

Fig. 5 compares the average overall reward, i.e., the overall weighted network utility averaged over $T=1000$ time slots, of the proposed strategy against all benchmark designs for various number of initial contents at the content server. The initial size of library ranges from $F=50$ to $F=500$, with the finite content library capacity set to be $F^{[\max ]}=F+50$. The proposed strategy has a better average overall reward 


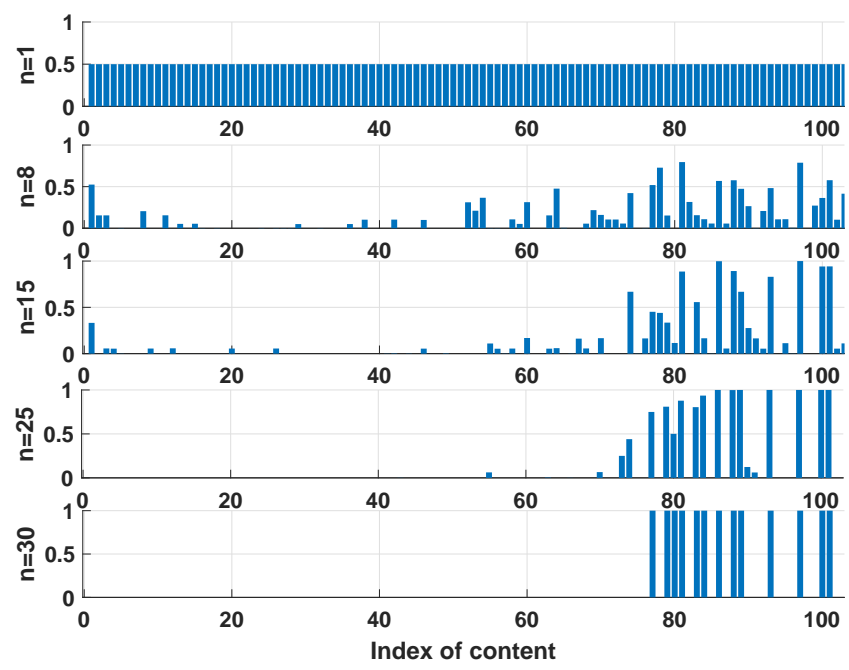

Fig. 4. Evolution of content placement policy via the CCE method.

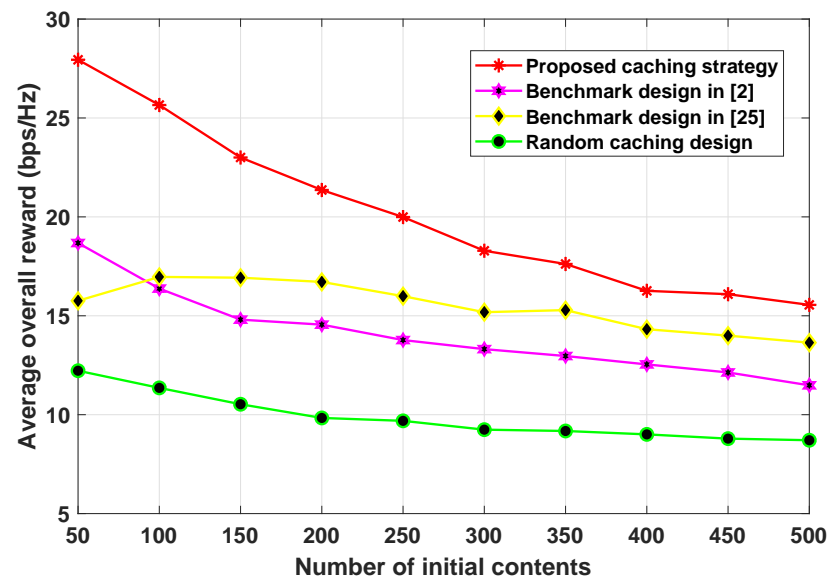

Fig. 5. Comparison of average overall reward for various number of initial contents.

as compared to the benchmark designs in [2] and [25], since neither the time-varying content popularity nor the nonstationary content library has been taken into consideration in their designs. Furthermore, one may conclude from Fig. 5 that the average performance of all strategies degrades with increasing number of initial contents, due to the fact that larger content library naturally results in more users' content requests being satisfied by the content server.

Fig. 6 depicts the average overall rewards of all strategies for various storage sizes, ranging from 5 to 35 , at the SBSs. As can be seen from Fig. 6, the performance of all strategies improves with the increasing capacity of the individual cache units at the SBSs. This is due to the fact that with larger caching space, the SBSs can cache more popular contents locally and reduce duplicate data transmission via avoiding retrieving data from the content server, and thus, offload more traffic from the content server to the edge. Moreover, it is worth noticing that the increasing capacity of cache units at the SBSs has less

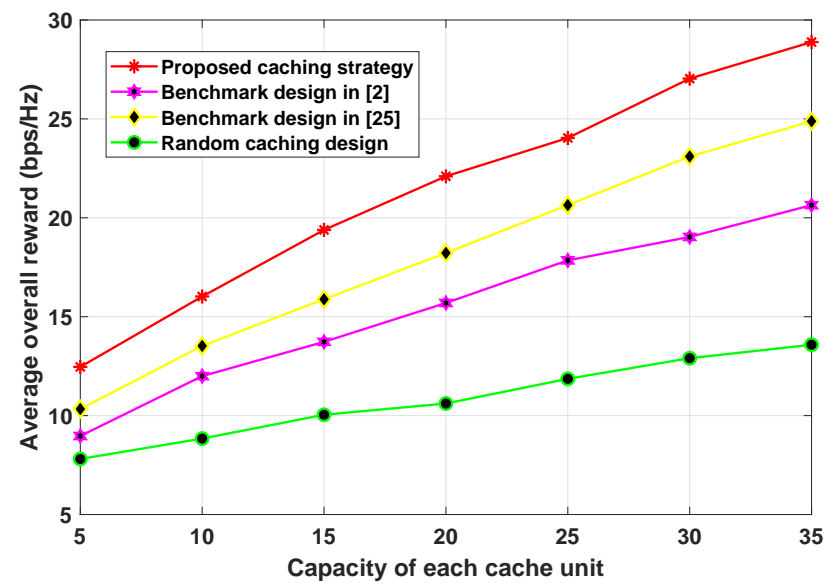

Fig. 6. Comparison of average overall reward for different capacity of individual cache units at the SBSs.

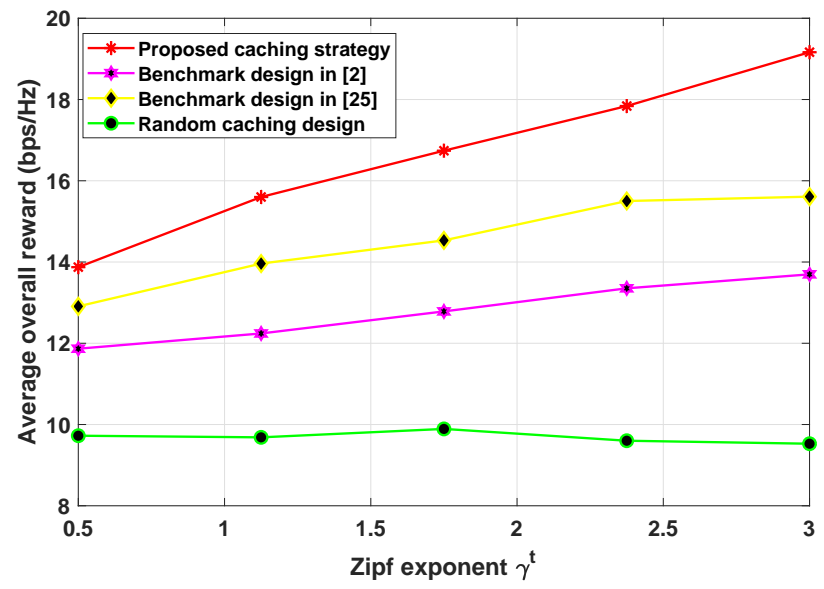

Fig. 7. Comparison of average overall reward for different content popularity variations.

impact on the random caching design as compared to that of the other strategies. The reason is that the former neglects the potentiality of the content caching coordination among SBSs and may have cached duplicate contents at different SBSs, hence wasted some of the limited caching space.

Fig. 7 presents the average overall rewards of all strategies for various actual content popularity variations, where the Zipf exponent $\gamma^{t}$ is set to be $\{0.5,1.125,1.75,2.375,3\}$, respectively. It is evident from Fig. 7 that both the proposed strategy and the learning based benchmark designs in [2] and [25] have improved performance with increasing value of $\gamma^{t}$, whilst less influence is observed for the random caching design under different values of $\gamma^{t}$. More specifically, when $\gamma^{t}$ is larger, the majority of the content demands of the users are occupied by fewer most frequently requested contents, hence is more favourable for the learning based content caching designs.

Fig. 8 shows the average overall rewards of all strategies for various number of emerged new contents $N_{f}^{[\text {new] }}$. One may conclude from the figure that though having better performance as compared to the random caching design, the 


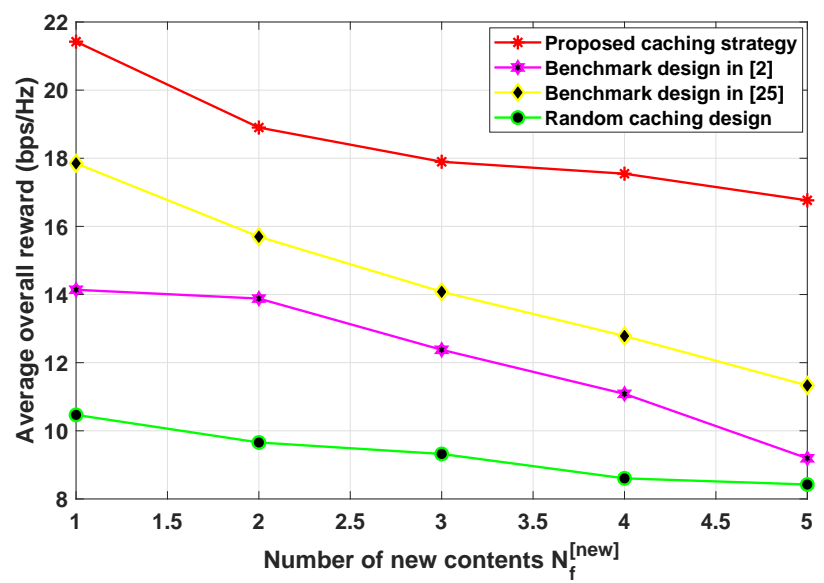

Fig. 8. Comparison of average overall reward for different number of new contents.

average overall rewards of the proposed strategy as well as the benchmark designs in [2] and [25] decrease with the increasing number of $N_{f}^{[\text {new }]}$. This is due to the fact that for a larger value of $N_{f}^{[\text {new] }}$, it is more challenging for the learning process to catch up with such rapid changes in users' content demands. However, as can be observed from the figure, the proposed strategy demonstrates its superiority in coping with the rapid variations of user demands, as compared to the benchmark designs in [2], [25]. On the contrary, the variations in $N_{f}^{[\text {new] }}$ have less impacts on the random caching design due to the fact that it fails to satisfy users' content requests for most of the time.

\section{CONCLUSION}

The joint cache placement and content delivery problem in small cell networks is studied in this paper, where the spatiotemporal dynamic content popularity is unknown a priori and the content library evolves over time. To account for the capacity-constrained cache units at the individual SBSs, content caching coordination among SBSs is adopted to improve the caching performance. To keep tracking the dynamic content library, a portion of each cache unit is assigned as the flexible cache that can be timely updated with the contents cached by users prior to the routine off-peak main cache update from the content server. Considering three phases of different time scales for the content delivery, the flexible cache update and the main cache update, the problem of interest is modelled as a reinforcement learning-assisted optimization problem and an user aided foresighted caching algorithm is proposed to maximize the long-term average weighted utility of the network. Simulation results confirm the superiority of the proposed foresighted strategy in achieving a significant performance improvement over various benchmark designs.

\section{REFERENCES}

[1] Cisco, "Cisco Visual Networking Index: Forecast and Methodology, 2016-2021”, White Paper, Sep. 2017.
[2] J. Song, M. Sheng, T. Q. S. Quek, C. Xu and X. Wang, "LearningBased Content Caching and Sharing for Wireless Networks," IEEE Transactions on Communications, vol. 65, no. 10, pp. 4309-4324, Oct. 2017.

[3] C. Fang, F. R. Yu, T. Huang, J. Liu and Y. Liu, "A Survey of Energy-Efficient Caching in Information-Centric Networking," IEEE Communications Magazine, vol. 52, no. 11, pp. 122-129, Nov. 2014.

[4] L. Li, G. Zhao and R. S. Blum, "A Survey of Caching Techniques in Cellular Networks Research Issues and Challenges in Content Placement and Delivery Strategies," IEEE Communications Surveys \& Tutorials, vol. 20, no. 3, pp. 1710-1732, Mar. 2018.

[5] E. Bastug, M. Bennis and M. Debbah, "Living on the Edge: The Role of Proactive Caching in 5G Wireless Networks," IEEE Communications Magazine, vol. 52, no. 8, pp. 82-89, Aug. 2014.

[6] Y. Zhu, G. Zheng, L. Wang, K-K. Wong and L. Zhao, "Content Placement in Cache-Enabled Sub-6 GHz and Millimeter-Wave MultiAntenna Dense Small Cell Networks," IEEE Transactions on Wireless Communications, vol. 17, no. 5, pp. 2843-2856, Oct. 2018.

[7] B. Blaszczyszyn and A. Giovanidis, "Optimal Geographic Caching in Cellular Networks," IEEE International Conference on Communications (ICC), pp. 3358-3363, Jun. 2015.

[8] J. Gu, W. Wang, A. Huang, H. Shan and Z. Zhang, "Distributed Cache Replacement for Caching-Enable Base Stations in Cellular Networks," IEEE International Conference on Communications (ICC), pp. 26482653, Jun. 2014

[9] M. Afshang, H. S. Dhillon and P. H. J. Chong, "Fundamentals of Cluster-Centric Content Placement in Cache-Enabled Device-to-Device Networks," IEEE Transactions on Communications, vol. 64, no. 6, pp. 2511-2526, Jun. 2016.

[10] R. Wang, X. Peng, J. Zhang and K. B. Letaief, "Mobility-Aware Caching for Content-Centric Wireless Networks: Modeling and Methodology," IEEE Communications Magazine, vol. 54, no. 8, pp. 77-83, Aug. 2016.

[11] B. Zhou, Y. Cui and M. Tao, "Stochastic Content-Centric Multicast Scheduling for Cache-Enabled Heterogeneous Cellular Networks," IEEE Transactions on Wireless Communications, vol. 15, no. 9, pp. 6284-6297, Sep. 2016

[12] Y. Cui and D. Jiang, "Analysis and Optimization of Caching and Multicasting in Large-Scale Cache-Enabled Heterogeneous Wireless Networks," IEEE Transactions on Wireless Communications, vol. 16 , no. 1, pp. 250-264, Jan. 2017.

[13] M. Tao, E. Chen, H. Zhou and W. Yu, "Content-Centric Sparse Multicast Beamforming for Cache-Enabled Cloud RAN," IEEE Transactions on Wireless Communications, vol. 15, no. 9, pp. 6118-6131, Sep. 2016.

[14] S-H. Park, O. Simeone and S. S. Shitz, "Joint Optimization of Cloud and Edge Processing for Fog Radio Access Networks," IEEE Transactions on Wireless Communications, vol. 15, no. 11, pp. 7621-7632, Nov. 2016.

[15] N. Karamchandani, U. Niesen, M. A. Maddah-Ali and S. N. Diggavi, "Hierarchical Coded Caching," IEEE Transactions on Information Theory, vol. 62, no. 6, pp. 3212-3229, Jun. 2016.

[16] Q. Yan, U. Parampalli, X. Tang and Q. Chen, "Online Coded Caching With Random Access," IEEE Communications Letters, vol. 21, no. 3, pp. 552-555, Mar. 2017.

[17] R. Pedarsani, M. A. Maddah-Ali and U. Niesen, "Online Coded Caching ", IEEE/ACM Transactions on Networking, vol. 24, no. 2, pp. 836-845, Apr. 2016.

[18] B. Chen, C. Yang and G. Wang, "High-Throughput Opportunistic Cooperative Device-to-Device Communications With Caching," IEEE Transactions on Vehicular Technology, vol. 66, no. 8, pp. 7527-7539, Aug. 2017.

[19] Z. Zhao, M. Peng, Z. Ding, W. Wang and H. Vincent Poor, "Cluster Content Caching: An Energy-Efficient Approach to Improve Quality of Service in Cloud Radio Access Networks," IEEE Journal on Selected Areas in Communications, vol. 34, no. 5, pp. 1207-1221, May 2016.

[20] B. Chen, C. Yang and Z. Xiong, "Optimal Caching and Scheduling for Cache-Enabled D2D Communications," IEEE Communications Letters, vol. 21, no. 5, pp. 1155-1158, May 2017.

[21] K. Poularakis, G. Iosifidis, V. Sourlas and L. Tassiulas, "Exploiting Caching and Multicast for 5G Wireless Networks," IEEE Transactions on Wireless Communications, vol.15, no.4, pp.2995-3007, Apr. 2016.

[22] C. Yang, Y. Yao, Z. Chen and B. Xia, "Analysis on Cache-Enabled Wireless Heterogeneous Networks," IEEE Transactions on Wireless Communications, vol. 15, no. 1, pp. 131-145, Jan. 2016

[23] E. Bastug, M. Bennis, E. Zeydan, M. A. Kader, I. A. Karatepe, A. S. Er and M. Debbah, "Big Data Meets Telcos: A Proactive Caching 
Perspective," Journal of Communications and Networks, vol. 17, no. 6, pp. 549-557, Dec. 2015.

[24] J. Kwak, Y. Kim, L. B. Le and S. Chong, "Hybrid Content Caching in 5G Wireless Networks: Cloud Versus Edge Caching," IEEE Transactions on Wireless Communications, vol. 17, no. 5, pp. 3030-3045, May 2018.

[25] P. Blasco and D. Gunduz, "Learning-based Optimization of Cache Content in a Small Cell Base Station," IEEE International Conference on Communications (ICC), pp. 1897-1903, Jun. 2014.

[26] A. Sadeghi and F. Sheikholeslami and G. B. Giannakis, "Optimal and Scalable Caching for 5G Using Reinforcement Learning of Space-Time Popularities," IEEE Journal of Selected Topics in Signal Processing , vol. 12, no. 1, pp. 180-190, Feb. 2018.

[27] B. N. Bharath, K. G. Nagananda, D. Gunduz and H. Vincent Poor, "A Learning-Based Approach to Caching in Heterogenous Small Cell Networks," IEEE Transactions on Communications, vol. 64, no. 4, pp. 1674-1686, Apr. 2016.

[28] S. Tamoor-ul-Hassan, S. Samarakoon, M. Bennis, M. Latva-aho and C. S. Hong, "Learning-Based Caching in Cloud-Aided Wireless Networks," IEEE Communications Letters, vol. 22, no. 1, pp. 137-140, Jan. 2018.

[29] M. Garetto, E. Leonardi, and S. Traverso, "Efficient Analysis of Caching Strategies under Dynamic Content Popularity," IEEE INFOCOM, Apr. 2015, pp. 2263-2271.

[30] C. R. Rao and V. Govindaraju, "Chapter 3 - The Cross-Entropy Method for Optimization", Machine Learning: Theory and Applications, Elsevier, 2013.

[31] M. Caserta, E. Quinonez Rico and A. Marquez Uribe, "A Cross Entropy Algorithm for the Knapsack Problem with Setups", Computers \& Operations Research, vol. 35, no. 1, pp. 241-252, Aug. 2008.

[32] M. Caserta and M. C. Nodar, "A Cross-Entropy Based Algorithm for Combinatorial Optimization Problems", European Journal of Operational Research, 2005.

[33] R. S. Sutton and A. G. Barto, "Reinforcement Learning: An Introduction," Cambridge MA, US: The MIT press, 2017.

[34] W. N. S. F. Wan Ariffin, X. Zhang and M. R. Nakhai, "Sparse Beamforming for Real-Time Resource Management and Energy Trading in Green C-RAN", IEEE Transactions on Smart Grid, vol. 8, no. 4, pp. 2022-2031, Jul. 2017.

[35] 3GPP, "TR 36.814 V9.2.0: Further Advancements for E-UTRA Physical Layer Spects (Release 9)," Available online: http://www.3gpp.org, Mar. 2017.

[36] D. Bouneffouf, R. Feraud, "Multi-armed Bandit Problem with Known Trend," arXiv:1508.07091, May 2017. 